\title{
SOLVING THE SCHRÖDINGER EQUATION
}

Has Everything Been Tried? 
This page intentionally left blank






\section{SOLVING THE
SCHRÖDINGER \\ SOLVING THE
SCHRÖDINGER $\underset{\text { Has Everthting Been Tried? }}{\mathrm{EQU}}$ EOUATION}

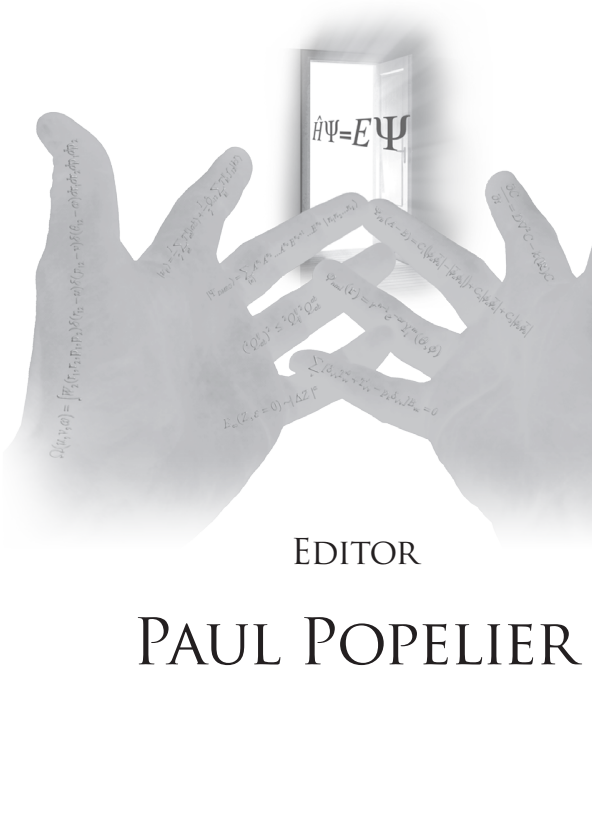

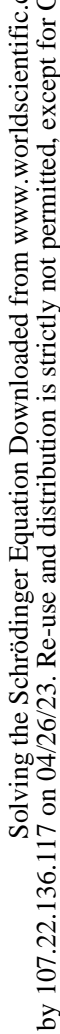




\section{Published by}

Imperial College Press

57 Shelton Street

Covent Garden

London WC2H 9HE

\section{Distributed by}

World Scientific Publishing Co. Pte. Ltd.

5 Toh Tuck Link, Singapore 596224

USA office: 27 Warren Street, Suite 401-402, Hackensack, NJ 07601

UK office: 57 Shelton Street, Covent Garden, London WC2H 9HE

\section{British Library Cataloguing-in-Publication Data}

A catalogue record for this book is available from the British Library.

\section{SOLVING THE SCHRÖDINGER EQUATION Has Everything Been Tried?}

Copyright $(92011$ by Imperial College Press

All rights reserved. This book, or parts thereof, may not be reproduced in any form or by any means, electronic or mechanical, including photocopying, recording or any information storage and retrieval system now known or to be invented, without written permission from the Publisher.

For photocopying of material in this volume, please pay a copying fee through the Copyright Clearance Center, Inc., 222 Rosewood Drive, Danvers, MA 01923, USA. In this case permission to photocopy is not required from the publisher.

ISBN-13 978-1-84816-724-7

ISBN-10 1-84816-724-5

Typeset by Stallion Press

Email: enquiries@stallionpress.com

Printed in Singapore. 
To D.P.B.

రั

逢

잉

(5)

.

s.

3

킁

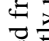

ㅇํㅇ

흘 
'The richest interactions occur between two almost identical but opposing constituents.' 


\section{Contents}

Preface $\quad$ Xv

1. Intracule Functional Theory 1

Deborah L. Crittenden and Peter M.W. Gill

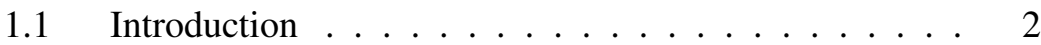

1.2 Intracules . . . . . . . . . . . . . 3

1.3 Electron Correlation Models . . . . . . . . . . . . 13

1.4 Dynamic and Static Correlation . . . . . . . . . . . 16

1.5 Dispersion Energies . . . . . . . . . . . . . . . 18

1.6 Future Prospects . . . . . . . . . . . . . . 21

Bibliography .................... 22

2. Explicitly Correlated Electronic Structure Theory 25

Frederick R. Manby

2.1 Introduction . . . . . . . . . . . 25

2.1.1 Basis-set expansions . . . . . . . . . . 25

2.2 F12 Theory . . . . . . . . . . . . . 28

$2.2 .1 \quad$ MP2-F12 . . . . . . . . . . . . . . 29

2.2.2 Explicitly correlated coupled-cluster theory ..................... 30

2.3 Five Thoughts for F12 Theory . . . . . . . . . . . . 31

2.3.1 Thought 1: Do we need (products of) virtuals? . . . . . . . . . . . . . 31

2.3.2 Thought 2: Are there better two-electron basis sets? . . . . . . . . . . . . 34 
2.3.3 Thought 3: Do we need the resolution of the identity?

2.3.4 Thought 4: Could we have explicit correlation for higher excitations?

2.3.5 Thought 5: Can we avoid three-electron errors in two-electron systems? . . . . . . . . . . . 39

2.4 Conclusions . . . . . . . . . . . . . . . 40

Bibliography ................... 40

3. Solving Problems with Strong Correlation Using the Density Matrix Renormalization Group (DMRG)

Garnet Kin-Lic Chan and Sandeep Sharma

3.1 The Problem of Strong Correlation . . . . . . . . . . 43

3.2 The Density Matrix Renormalization Group

Wavefunction ................ 46

3.3 Locality and Entanglement in the DMRG . . . . . . . . 47

3.4 Other Properties of the DMRG . . . . . . . . . . . 50

3.5 Relation to the Renormalization Group . . . . . . . . 51

3.6 Dynamic Correlation - the Role of Canonical

Transformations ................ 53

3.7 What Can the DMRG Do? A Brief History . . . . . . . 54

3.8 The Future: Higher Dimensional Analogues . . . . . . 57

Bibliography ..................... 59

4. Reduced-Density-Matrix Theory for Many-electron Correlation 61 David A. Mazziotti

4.1 Introduction . . . . . . . . . . . . . . 62

4.2 Variational 2-RDM Method ............ 63

4.2.1 Energy as a 2-RDM functional . . . . . . . 63

4.2.2 Positivity conditions . . . . . . . . . . . 64

4.2.3 Semidefinite programming . . . . . . . . . 67

4.2.4 Applications . . . . . . . . . . . . . . . 69

4.3 Contracted Schrödinger Theory . . . . . . . . . . . . 73

4.3.1 ACSE and cumulant reconstruction . . . . . 74

4.3.2 Solving the ACSE for ground and excited states . . . . . . . . . . . 75

4.3.3 Applications . . . . . . . . . . . . 77

4.4 Parametric 2-RDM Method . . . . . . . . . . . . 80

4.4.1 Parametrization of the 2-RDM . . . . . . 81 
4.4.2 Applications . . . . . . . . . . . . 83

4.5 Looking Ahead . . . . . . . . . . . . . . 85

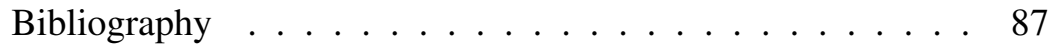

5. Finite Size Scaling for Criticality of the Schrödinger Equation 91 Sabre Kais

5.1 Introduction . . . . . . . . . . . . . 92

5.2 Criticality for Large-dimensional Models . . . . . . . . 93

5.3 Finite Size Scaling: A Brief History . . . . . . . . . . . 95

5.4 Finite Size Scaling for the Schrödinger Equation . . . . 97

5.5 The Hulthen Potential . . . . . . . . . . . . . . 100

5.5.1 Analytical solution . . . . . . . . . . . . 100

5.5.2 Basis set expansion . . . . . . . . . . . 101

5.5.3 Finite element method . . . . . . . . . . . 101

5.5.4 Finite size scaling results . . . . . . . . 102

5.6 Finite Size Scaling and Criticality

of M-electron Atoms . . . . . . . . . . . . . . . . 105

5.7 Conclusions . . . . . . . . . . . . . . 107

Bibliography .................... 108

6. The Generalized Sturmian Method 111

James Avery and John Avery

6.1 Description of the Method . . . . . . . . . . . . 111

6.1.1 The introduction of Sturmians

into quantum theory . . . . . . . . . . 111

6.1.2 Generalized Sturmians . . . . . . . . . . . . . 114

6.1.3 The generalized Sturmian method applied to atoms . . . . . . . . . . . . 117

6.1.4 Goscinskian configurations . . . . . . . 118

6.1.5 Goscinskian secular equations for atoms and atomic ions . . . . . . . . . 120

6.2 Advantages: Some Illustrative Examples . . . . . . . . 120

6.2.1 The large- $Z$ approximation: restriction of the basis set to an $\mathcal{R}$-block . . . . . . . . 121

6.2.2 Validity of the large- $\boldsymbol{Z}$ approximation . . . . . 126

6.2.3 Core ionization energies . . . . . . . . . . . 129

6.3 Limitations of the Method; Prospects for the Future . . 130

6.3.1 Can the generalized Sturmian method be applied to $N$-electron molecules? . . . . . . 133 
6.4 Discussion . . . . . . . . . . . . . . . 137

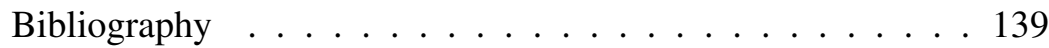

7. Slater-Type Orbital Basis Sets: Reliable and Rapid Solution of the Schrödinger Equation for Accurate Molecular Properties

Philip E. Hoggan

7.1 Introduction . . . . . . . . . . . . . . . 142

7.1.1 Context of this chapter . . . . . . . . . . 142

7.1.2 Atomic orbitals . . . . . . . . . . . . . . . 144

7.1.3 Problems to be solved when using

Slater-type orbitals . . . . . . . . . . . 145

7.1.4 Strategy for Slater basis sets . . . . . . . . . 147

7.2 Some Dates: The Story So Far of Slater-Type

Orbitals . . . . . . . . . . . . . . . . . . . 148

7.3 Computer Programs Using Slater-Type Orbitals . . . . 149

7.3.1 Numerical grid methods . . . . . . . . . . . . 151

7.3.2 Configuration interaction . . . . . . . . 151

7.4 Slater Orbitals and Gaussian Orbitals . . . . . . . . . 151

7.5 Types of Exponentially Decaying Orbitals, Based on Eigenfunctions for One-Electron Atoms . . . . . . . 154

7.5.1 Orbitals which are linear combinations of Slater-type orbitals . . . . . . . . . 155

7.6 Types of Integral Over Slater Orbitals . . . . . . . . . 156

7.6.1 One-electron integrals . . . . . . . . . . . 156

7.6.2 Two-electron integrals . . . . . . . . . . . 157

7.6.3 Three- and four-electron integrals . . . . . . 158

7.7 Integration Methods in the Literature . . . . . . . . . 158

7.7.1 Single-center expansion . . . . . . . . . . . . 159

7.7.2 Gaussian expansion . . . . . . . . . . . . 160

7.7.3 Gaussian transform method . . . . . . . . . 160

7.7.4 Fourier-transform method . . . . . . . . . 160

7.7 .5 Use of Sturmians . . . . . . . . . . . . . . 161

7.7.6 Elliptic coordinate method . . . . . . . . . . 161

7.7.7 Monte Carlo integration . . . . . . . . . . . 162

7.8 General Two-Electron Exponential Type Orbital Integrals in Poly-Atomics Without Orbital Translations 163 7.8.1 Introduction . . . . . . . . . . 163

7.8 .2 Basis sets . . . . . . . . . . . . . . 164 
7.8.3 Programming strategy . . . . . . . . . . . 164

7.8.4 Avoiding ETO translations for two-electron integrals over three- and four-centers. . . . . . 165

7.8.5 Numerical results of Coulomb resolutions: efficiency . . . . . . . . . . . . 167

7.8.6 Perspectives and conclusions . . . . . . . . . . 169

7.8.7 Angular momentum relations . . . . . . . . 171

7.9 When are Slater-Type Orbitals Advantageous?

Some Applications . . . . . . . . . . . . . . . . . . . . 171

7.9.1 The NMR nuclear shielding tensor . . . . . . 171

7.9.2 Explicitly correlated methods for molecules . 176

7.9.3 Trial wave-functions for quantum Monte Carlo simulations over STO . . . . . . . . . . 178

7.10 Highly Accurate Calculations Using STOs . . . . . . . 181

7.11 Closing Remarks . . . . . . . . . . . . . . 181

7.12 Appendix A: How STOs were Translated: Products on Two Atoms . . . . . . . . . . . . . . . . . . . . 183

7.12 .1 Review of BCLFs . . . . . . . . . . . . . 184

7.13 Appendix B: Brief Time-Line of Events in Molecular Work Over Slater-Type Orbitals to Date . . . . . . . 186

7.14 Appendix C: Main Results of Podolanski's Paper of 1931 with Additional Comments . . . . . . . . . . . 187

7.15 Appendix D: Potentials and Auxiliary Overlaps for Coulomb Resolution . . . . . . . . . . . . . . . . . 189

7.16 Appendix E: Analysis of Nuclear Dipole Integrals for NMR in a Slater Basis . . . . . . . . . . . . . . . 191

Acknowledgements . . . . . . . . . . . . . . 193

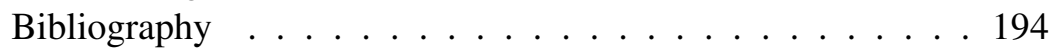

8. Modern Ab Initio Valence Bond Methods 201

Philippe C. Hiberty and Sason Shaik

8.1 Basic Principles and Survey of Modern Methods . . . . 202

8.1.1 VB vs. MO wave functions in the two-electron/ two-center case . . . . . . . . . . . 202

8.1.2 Writing VB functions beyond the two-electron/ two-center case . . . . . . . . . . . . . 205

8.1.3 Some landmark improvements of the early VB method . . . . . . . . . . . . . . . . 206 
8.2 Strengths of the Valence Bond Approach . . . . . . . . 211

8.2.1 Interpretability combined with accuracy of the wave functions . . . . . . . . . . 211

8.2.2 A simple solution to the symmetry dilemma . 213

8.2.3 Calculations of diabatic energy curves along a reaction coordinate . . . . . . . . . . 214

8.2.4 Quantitative evaluation of common chemical paradigms . . . . . . . . . . 218

8.3 Present Capabilities and Expected Improvements . . . . 224

8.3.1 Evaluation of Hamiltonian matrix elements . . 224

8.3.2 Direct VBSCF/BOVB algorithm . . . . . . . . 224

8.3.3 Current calculations of medium-sized molecular systems . . . . . . . . . . 225

8.3.4 Mixed Valence Bond - Quantum Monte Carlo methods . . . . . . . . . . . . . 225

8.3.5 Prospective . . . . . . . . . . . . 226

8.4 Concluding Remarks . . . . . . . . . . . . . . 228

8.5 Appendix A: The Myth of "VB failures" . . . . . . . . 229

8.6 Appendix B: Some Available VB

Software Packages . . . . . . . . . . . . . . 230

8.6.1 The XMVB program . . . . . . . . . . . 230

8.6 .2 The TURTLE software . . . . . . . . . . 230

8.6.3 The VB2000 software . . . . . . . . . . 230

8.6.4 The CRUNCH software . . . . . . . . . . . 231

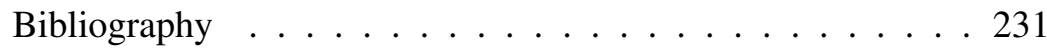

9. Quantum Monte Carlo Approaches for Tackling

Electronic Correlation

Massimo Mella and Gabriele Morosi

9.1 Introduction . . . . . . . . . . . . . 238

9.2 Variational Monte Carlo (VMC): A Possible

Way Toward Explicitly Correlated Electronic

Wave Functions . . . . . . . . . . . . . . . . . 239

9.2.1 Numerical integrals in VMC . . . . . . . . . . 241

9.2.2 Optimization of trial wave functions . . . . . . 247

9.2.3 Analytical forms for trial wave functions $\Psi_{T} \ldots \ldots \ldots$. . . . . . . . 252

9.3 Diffusion Monte Carlo: How to Extract the Best

Information from Inaccurate Wave Functions . . . . . . 254 
9.3.1 Generalities . . . . . . . . . . . 254

9.3.2 Improved projectors . . . . . . . . . . . . 258

9.3.3 DMC, state symmetry and excited states . . . 259

9.4 Computing Observables Different from State Energy . . 261

9.4.1 Exact calculation of position dependent observables . . . . . . . . . . . . 261

9.4.2 Calculation of atomic forces in VMC/DMC . . 262

9.4.3 Computing the expectation value of ultra-local operators: electron and spin density on nuclei . . . . . . . . . . . . . . . . 264

9.5 Conclusions . . . . . . . . . . . . . . 266

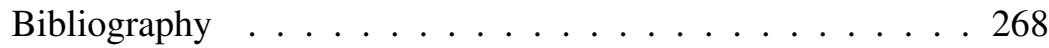

10. Solving the Schrödinger Equation on Real-Space Grids and with Random Walks

Thomas L. Beck and Joel H. Dedrick

10.1 Introduction . . . . . . . . . . . . . . . . 272

10.2 Solving the Schrödinger Equation Using Grids in Real Space . . . . . . . . . . . . . . 275

10.2.1 Basics of grid methods . . . . . . . . . . . 275

10.2.2 Multiscale (multigrid) approaches . . . . . . . 279

10.3 New Ways of Thinking about Large-Scale

Solutions . . . . . . . . . . . . . . . . . . 282

10.3.1 An encounter with Silicon valley _. . . . . 283

10.3.2 The Borg-ing of computation . . . . . . . . 283

10.3.3 The world's least efficient computer (yours) . . 283

10.3.4 The world's most efficient computer (also yours) . . . . . . . . . . . . . 284

10.3.5 The end of a 20-year free ride . . . . . . . 285

10.3.6 Can we change sides? . . . . . . . . . . 286

10.3.7 Algorithm desiderata for the massively parallel future . . . . . . . . . . . . . 286

10.3.8 What are we looking for? . . . . . . . . . 288

10.4 Random Walks for Solving the Schrödinger

Equation . . . . . . . . . . . . . . . . 292

10.4.1 Traditional diffusion quantum Monte Carlo . . 292

10.4.2 Another angle . . . . . . . . . . . . . . 296

10.4.3 Stochastic differential equations and the Feynman-Kac approach . . . . . . . . . . 298 
10.4.4 Obtaining $\mathrm{E}(\mathrm{x}, \mathrm{y}) ? \ldots 302$

10.4.5 A pipe dream . . . . . . . . . . . . . 305

10.5 Summary . . . . . . . . . . . . . . 306

Bibliography ................. . . 307

11. Changes in Dense Linear Algebra Kernels: Decades-

Long Perspective

Piotr Luszczek, Jakub Kurzak, and Jack Dongarra

11.1 The Schrödinger Connection _. . . . . . . . . . . 313

11.2 A Stroll Down the Memory Lane . . . . . . . . . . . . 315

11.3 A Decompositional Approach . . . . . . . . . . . . 318

11.4 Vector Processors . . . . . . . . . . . . . . . . . 319

11.5 RISC Processors . . . . . . . . . . . . . . . . . . 322

11.6 Clusters . . . . . . . . . . . . . . . 325

11.7 Multicore Processors . . . . . . . . . . . . . 332

11.8 Multicore Processors Redux . . . . . . . . . . . . . . . 334

11.9 Error Analysis and Operation Count . . . . . . . . . . 338

11.10 Future Directions for Research and Hardware Design . 339

Bibliography ................... 341

Index 


\section{Preface}

In the first of his four lectures on wave mechanics, Schrödinger wrote:

Substituting from (12) and (8) in (10) and replacing p by $\psi(\ldots)$ we obtain

$$
\nabla^{2} \psi+\frac{8 \pi^{2} m}{h^{2}}(E-V) \psi=0
$$

(...) A simplification in the problem of the "mechanical waves" consists in the absence of boundary conditions. I thought the latter simplification fatal when I first attacked these equations. Being insufficiently versed in mathematics, I could not imagine how proper vibration frequencies could appear without boundary conditions. Later on I recognised that the more complicated form of the coefficients (i.e. the appearance of $V(x, y, z)$ ) takes charge, so to speak, of what is ordinarily brought about by boundary conditions, namely, the selection of definite values of $E$.

(Dr. Erwin Schrödinger - Four Lectures on Wave Mechanics. Delivered at the Royal Institution, London, on 5, 7, and 14 March, 1928)

Here we are: equation number 13 arrived in 'Knowledge Space' and is there to stay. A simple looking equation, one of many in Schrödinger's paper, and one that can be written even simpler as $H \psi=E \psi$. Although short in appearance, this equation is immensely powerful. It governs the behaviour and properties of all matter at ambient conditions. It is, therefore, the mother equation of chemistry. Who could have thought, though, that some 85 years later, people are still thinking about solving it faster and more accurately? Are the standard methods to solve the molecular electronic Schrödinger equation, which are implemented in popular commercial $a b$ 
initio programs, the best we can achieve? Or is there a new and powerful idea lurking at the surface of 'Knowledge Space', which leads to a better method, more accurate and faster, and independent from (chemical) experiment? Is this new idea based on the combination of two or more existing ideas? This book asks these difficult and ambitious questions to its contributing authors and to the reader.

This book invited its authors to elucidate the non-standard method that they specialised in, explain its strength and weakness, and then speculate about what is needed to widen the application radius of the method. Actually achieving this may take years and involve several people. This book hopes to inspire readers and researchers by putting non-standard approaches together in one place. I believe that this has never been done. The format and style in which the chapters are written should make it possible to read the whole book through. It should be emphasised that this text was not designed as a review. Instead, it is meant to be a collection of personal accounts capturing the aspiration and perhaps frustration of experts of non-standard methods.

So, what can we learn from Schrödinger's aforementioned quote, other than that it is good to catch up with a sufficient amount of mathematics (or spend Christmas holidays frolicking with an old flame in a mountain resort, where he discovered his equation)? One lesson is to trust the potential of an idea, often based on an analogy or a vivid picture. In fact, in Schrödinger's case this was the symbolic proportion:

\section{Ordinary mechanics : Wave mechanics}

\section{$=$ Geometrical optics : Undulatory optics.}

His 'derivation' of Eq. (13), developed in the pages leading up to the excerpt above, is based on this analogy. Schrödinger could describe what his new 'wave mechanics' would look like based on this intuitive analogy. As he worked out the maths behind this intuitive development he panicked for a moment, due to his lack of mathematical knowledge ${ }^{1}$. Fortunately, he ended up with an equation that worked. Moreover, Schrödinger presented quantum mechanics with a completely new formalism, dual to the older matrix mechanics, which Heisenberg had proposed. The latter, and other members of the Copenhagen clan, did not like wave mechanics much: it was too intuitive and not as elegant and 'deep' as matrix mechanics. Matrices were of course wonderfully abstract mathematical entities to the theoretical physicists of that generation. However, this abstraction did not endow matrix mechanics with any authority over wave mechanics unless

\footnotetext{
${ }^{1}$ Quantum Mechanics textbooks typically gloss over this concern. Is there something deeper in the reassurance of $V(x, y, z)$ acting as a boundary condition?
} 
by a vague sense of mysterious elegance. To the contrary, matrix mechanics has remained rather useless to chemistry; solving the hydrogen atom by this method took Pauli pages and pages of calculation.

Returning to trusting the potential of an idea, this trust was also present in the development of Valence Bond and Molecular Orbital theories. Both describe the same reality, but from diametrically opposed views. Of utmost importance here is the confidence that a visual and intuitive approach to a problem, or even thinking about it in words, can be an excellent starting point for a 'derivation' that leads to a new and powerful computational scheme. It would be unnatural to derive Valence Bond theory from Molecular Bond theory, or vice versa. Of course, one can formally link the two, as one can do with matrix mechanics and wave mechanics. The point is that equivalent but very different approaches originate from different ideas, each based on an insight originally conceived as an image or stated in words. Armed with this confidence in the potential of a fresh idea one should ask: have we been imaginative enough in our intuition, pictures and words, when trying to solve the Schrödinger equation?

Speaking of imagination, one cannot emphasise enough the importance of thinking outside the box or what I call 'let go' thinking. One could object to taking a square root of a negative number or, instead, embrace this idea, give the result a name, work with it and derive the full consequences. Algebra has been helped tremendously by the 'let go' concept of an imaginary number. Of course, thinking outside the box is not a guarantee for success or a breakthrough. Quaternions, for example, are even more imaginative than imaginary numbers but they have not lived up to the rich expectations that their originator, Hamilton, fostered. Even less enthused in terms of application or deep insight should one be when it comes to octonions, or non-integer derivatives.

Still, the very attitude of 'let go' is powerful when it comes to letting go of a deeply ingrained assumption. An assumption that one cannot justify or that one does not even realise one is making. A nice example can be found in the planetary motion of our solar system. The Greeks revered symmetry, to the point of adhering religious connotations to it. Symmetry had a transcendental or even divine air. Invariance or constancy can also be regarded as a form of symmetry. Surely the motion of celestial bodies must be governed by symmetry, given the divine character of 'heavenly objects'. Ptolemaic astronomy (that had entered Greek astronomy) is a scheme in which a planet moves in a circle and at constant motion, both, indeed, signatures of symmetry. In Copernicus' time this model became horribly complicated in order to explain the large number of observations gathered 
by the Arabs since the ancient Greek times. The Ptolemaic theory needed 77 circles to describe the motion of the sun, moon, and the five planets then known. Kepler broke with the tradition of 2,000 years, that circles must be used to describe heavenly motions. He showed that a single ellipse would do. An ellipse is not as symmetrical as a circle, and therefore not as 'heavenly'. A circle can be seen as an ellipse in which the two foci have collapsed to one (and hence the two radii as well). Actually, in a deeper way, an ellipse is more heavenly than a circle because it captures Nature in a minimal model. In such a model there is no need for corrections within corrections. All falls in place by letting go of the constraint that a planet must move in a circular orbit. I now wonder where our 'circles' are in quantum chemistry. Which constraints are we holding on to?

This book invites its authors and the readers to abandon the usual lines of thoughts and presumptions that we do not perhaps realise we are making. The most powerful theories are minimal, not simple. 'Simple' means that we impose an unwarranted constraint onto what we are trying to explain. 'Minimal' means that we discovered the most essential, but unconstrained concept that governs the observed data of interest. This economy of principle or assumption always pays off, but obtaining a minimal theory requires much imagination and audacity.

Returning to quantum chemistry, one may have the impression that the only truly predictive computational schemes are built on brute force foundations. The core idea behind configuration interaction is brute force in nature. The explosion in computational work it leads to warrants clever but inevitably approximate computational schemes. This is only vaguely reminiscent of the combinatorial explosion encountered in the calculation of a determinant of a large matrix by the definition of an alternating sum of permuted terms. This idea leads to intractable calculations for a matrix as small as a $30 \times 30$ matrix, for example. Yet calculating such a determinant is perfectly feasible with LU decomposition in most reasonable times. This is where the power of the idea makes apparently impossible tasks possible after all. Then we can ask again: why can we not think of a method to solve the Schrödinger equation of a protein beyond Coupled-Cluster Singles and Doubles (Triples)/ Complete Basis Set (CCSD(T)/CBS) quality in a few seconds?

Regarding the content of this book, one can see that there are eleven chapters, covering ten ideas (or methods) not prevalent in current mainstream quantum chemistry. Unfortunately, some methods are not included, due to a lack of available authors. Otherwise, there would have been extra chapters on Bohmian mechanics, the series solution method, the 
pseudospectral method and the iterative configuration interaction method. Another, perhaps more striking omission is that of Density Functional Theory (DFT). The only chapter on DFT in the spirit of this book would have to explain fresh ideas on finding the ultimate exact functional. Much has been written on the theoretical conditions that this functional should obey. Ironically, the most successful functionals in current use seem to violate one of more of these conditions. If that is, to some readers, a reason to have included a fresh chapter on DFT then I apologise. If this book ever reaches a second edition a chapter on DFT should be considered, as well as a recorded roundtable discussion on the various methods presented in it, as in the book 'Quo vadis Quantum Mechanics?'

I am very grateful, indeed, for the hard work and enthusiasm that the authors have put in this book. Without them, this book would not be possible. Thank you all and may you not buckle under the pressures imposed by ignorant politicians, policy-makers and managers, serving an ever emptier and short-term society. Being driven by good old fashioned curiosity alone should be respectable again.

Finally, I'd like to quote from a book I recently read. At the very end of his book entitled 'The Trouble with Physics' on the rise of string theory, the fall of a science and what comes next, Lee Smolin concludes, '... I am going to turn off the phone and the BlackBerry, put on [... my favourite music ...], erase the blackboard, get out some good chalk, open a new notebook, take out my favourite pen, sit down, and start thinking.' One can query if modern electronic structure theory is in the same situation as this part of physics, which pursues a grand unified theory. But even if the situation is vaguely similar, many of us should buy some good chalk also. I only hope that reading this book in full will help, as well as the idea exchange that it hopes to catalyse, one day ...

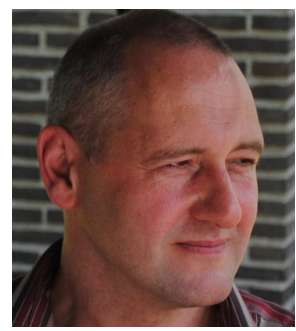

Paul Popelier Manchester, 11 September 2010 